\title{
A Fatal Congenital Defect Associated with a Unique Chromosome Abnormality
}

\author{
A. D. BAIN and I. K. GAULD \\ From the Department of Pathology, Royal Hospital for Sick Children and the University of Edinburgh
}

The foetus described in this communication showed unusual pathological features and an unusual abnormal karyotype. The foetus was sent to us for routine necropsy, diagnosed as macerated, with haemolytic disease. In view of the external appearance, chromosome studies were carried out.

\section{Case Report}

Maternal History. The father and mother were both aged 39 and were first cousins. There was no maternal history of radiation. This was the seventh pregnancy. She had had a previous stillbirth, but all babies with the exception of the present had been born at home. The 5 children, aged from 3 to 16 years, were alive and well. A normal live child was born to the parents 19 months after the birth of this abnormal foetus.

Necropsy Findings. A male foetus, weighing $900 \mathrm{~g}$., was born after a gestation of 29 weeks. The foetus showed a striking abnormality in the external appearance. The skin of the entire body was extremely lax, and subcutaneous oedema was evident in localized areas, particularly over the hands and feet. Tears involving the entire thickness of the skin were present on the side of the neck, in the groin, and in the scalp. In the scalp the tear, fully $6 \mathrm{~cm}$. in length, involved the entire thickness. To the inexperienced, the external appearance might well have suggested maceration. Other external abnormalities were present; the facial appearance was peculiar with flattening of the nose and oddly-shaped eyes; the significance of this in view of the skin abnormality remained indeterminate. The ears, particularly the left, were abnormal. There was an unusually higharched palate. The fingers showed a flexion deformity with the index finger flexed obliquely over the palm and overlapped by the middle and ring fingers. The lower limbs were rigid and fixed in extension with no possible movement at the knee joint. The feet were grossly abnormal, one showing a varus and the other a valgus type deformity; they showed a flattening probably accentuated by the laxity of the skin and subcutaneous oedema; both great toes were undoubtedly short. The

Received January 25, 1966. brain and meninges were apparently normal; there was no falx defect and both olfactory nerves were present. The heart and lungs were normal; detailed dissection excluded the presence of any congenital cardiac defect. In the abdomen there was an excess of free fluid in the peritoneal sac, but no abnormality was noted in any of the organs. There was no defect of the kidneys or urinary tract.

On histological examination the nephrogenic zone of the kidneys was much narrower than the birthweight would have indicated. No histological abnormality was noted in any of the internal organs or in the testes. The skin, however, showed changes that were uniformly present in sections taken from various areas. Histological changes were evident in the dermis, which was unusually cellular and comprised mainly of fibroblasts with little, if any, collagen or elastic tissue (Fig. I). The epidermis appeared normal with normal keratinization for the maturity of the foetus.

The placenta was not available for pathological examination. It was reported to be large. The portion of umbilical cord still attached to the baby possessed three vessels.

Chromosome Analysis. Colchicine-inhibited metaphase preparations were obtained from cultures of thymus and spleen using a modification of our original technique (Bain and Gauld, 1964). The splenic culture yielded considerably more cells in mitosis than did that from the thymus. 30 cells from each culture were examined. The chromosome number in every instance was 47. On analysis the cells showed a small extra acrocentric chromosome, a chromosome missing in the 19/20 group, and an extra metacentric chromosome slightly larger than a No. I6 (Fig. 2). These findings were later confirmed in explant cultures of fibroblasts derived from lung.

Chromosome preparations were obtained from the blood lymphocytes of father and mother. No apparent abnormality was present, and neither parent showed anything to suggest a polymorphic No. 16 chromosome.

\section{Discussion}

A foetus showing abnormalities such as this is, in our experience, unusual. With the exception of 


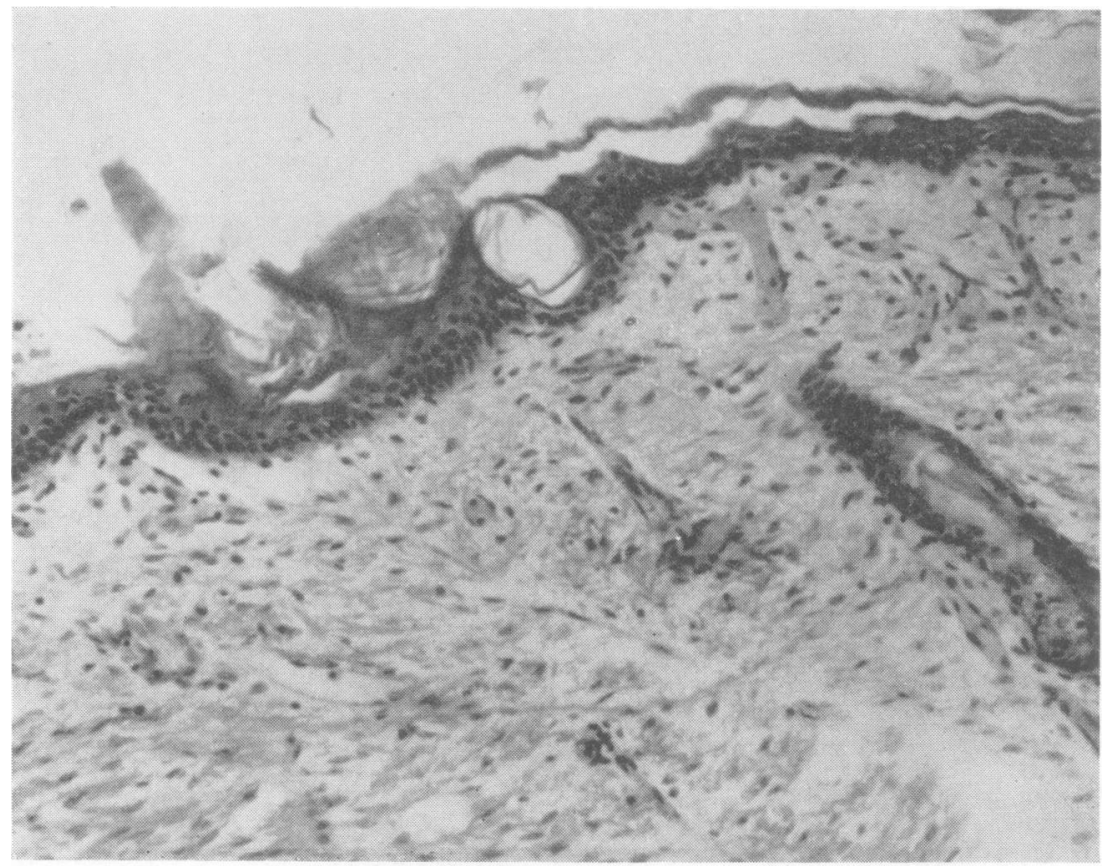

FIG. I. Section of skin showing fibroblastic nature of the dermis. (H. and E. $\times$ roo.)

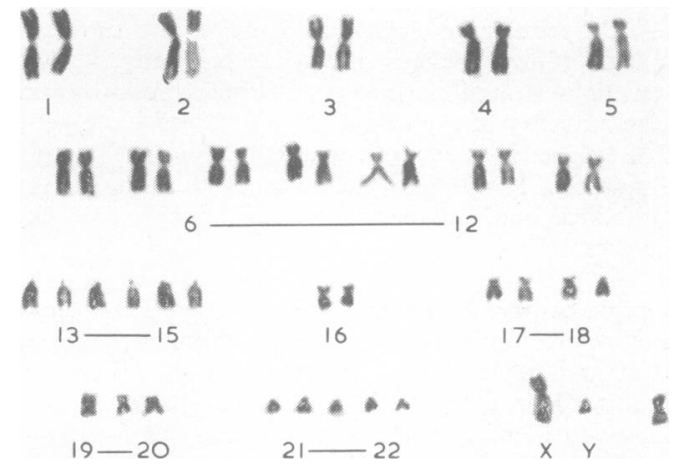

FIG. 2. Chromosomes from splenic culture. $(X$ 1200. $)$ The abnormal metacentric chromosome is placed at the bottom right.

the skin defect, the external abnormalities were similar to those that have been described in association with abnormalities of $13 / 15$ or $17 / 18$ groups of chromosomes. However, the absence of abnormalities in the heart, brain, or kidneys would be an unusual finding if these chromosomes were involved.

A defect of the skin, such as was present in this case, would inevitably prove fatal at birth. The defect, which clinically might be described as a severe form of cutis laxa, lay in an abnormality of the dermis. A comparison was made with the skin of as foetus of the same maturity as the present case. The maturity was estimated on the development of

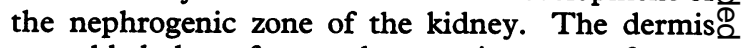
resembled that of a much more immature foetus, $\overrightarrow{\vec{B}}$ and histologically it might not be too speculative to 3 consider this abnormality as a maturation defect.

We feel that any classification of a karyotype suchọ. as this would be at present inadvisable and any hypothesis as to its origin too speculative. It willso therefore, be of interest if other cases similar to this are encountered.

\section{Summary}

A foetus is described showing multiple abnormalities and in particular a severe dermal defect. The abnormal karyotype has not been classified, $\frac{D}{O}$ and to the present appears to be a unique occurrence.

This work was supported by a grant from the Distillers Company Ltd. to the University of Edinburgh, and by the Board of Management, Royal Hospital for $\omega$ Sick Children, Edinburgh. We are grateful to Dr. W. Court-Brown, Miss P. Jacobs, and Dr. D. Harnden for their co-operation.

\section{REFERENCE}

Bain, A. D., and Gauld, I. K. (1964). The use of thymus and spleen in the demonstration of chromosomes postmortem in foetuses and infants. Brit. F. exp. Path., 45, 530. 